COVID-19 related restrictions have impacted the dynamics of romantic relationships, with many cohabiting partners spending more time together and non-cohabiting partners much less. We explored qualitatively, the vulnerabilities (characteristics that decreased resilience) and stressors that impacted intimate relationships following the initial COVID19 lockdown.

45 semi-structured interviews were undertaken with participants who had completed a national web-panel survey (NatsalCOVID) and agreed to follow-up. Here we draw on the accounts of 19 participants in steady relationships who reported relationship difficulties. Analysis drew on Karney and Bradbury's 'Vulnerability-stress-adaptation' model.

The sample comprised 12 women and 7 men, 13 were living with their partner and 6 were not. Participant's preexisting attachment, coping, and communication styles shaped their susceptibility to relationship difficulties. The stress of COVID-19, amplified by financial strain and health issues, affected couple's ability to adapt. In live-in relationships, childcare, divisions of housework, and a lack of space in which to unwind and escape from negative behaviours intensified pressures on relationship quality. One participant described these in the context of a violent relationship that worsened during lockdown, which she had managed to leave. Participants who did not live with their partners described struggling with phone/digital communication, physical distance, and a lack of certainty in the future of their relationship. In adapting to 'pandemic life', tensions arose over how much time to spend together. Those in non-cohabiting relationships were torn between balancing risks of COVID-19 against those of not seeing each other, with many reporting feeling they had placed their relationship on hold. For some, their sex life improved their adaptation, while for others it was a further source of stress.

Understanding how existing vulnerabilities interact with a stressful event to shape adaptive processes in couples' relationships might provide insights for counsellors and healthcare providers to better support couples through COVID-19.

\section{P289 A COST-NEUTRAL RAPID STI SERVICE IMPLEMENTATION PROVIDING THE RIGHT TREATMENT AT THE RIGHT TIME TO IMPROVE PATIENT EXPERIENCE AND OUTCOMES}

\begin{abstract}
1,2 J Boylan*, ${ }^{3}$ S Sundar, ${ }^{1} \mathrm{R}$ Gardiner, ${ }^{4} \mathrm{P}$ Muir, ${ }^{4} \mathrm{~J}$ Steer, ${ }^{4} \mathrm{R}$ Hopes, ${ }^{1} \mathrm{H}$ Wheeler, ${ }^{1} \mathrm{M}$ Clarke, ${ }^{1} \mathrm{M}$ Crofts, ${ }^{5,3} \mathrm{H}$ McLeod, ${ }^{3} \mathrm{G}$ Myring, ${ }^{1,3} \mathrm{P}$ Horner. ${ }^{1}$ Unity Sexual Health, University Hospitals Bristol and Weston NHS Foundation Trust, Bristol, UKi ${ }^{2}$ Liverpool University Hospitals NHS Foundation Trust, Liverpool, UK; ${ }^{3}$ Population Health Sciences, Bristol Medical School, University of Bristol, Bristol, UK; ${ }^{4}$ PHE South West Regional Laboratory National Infection Service, Bristol, UK; ${ }^{5}$ The National Institute for Health Research Applied Research Collaboration West (NIHR ARC West), Bristol, UK
\end{abstract}

10.1136/sextrans-2021-sti.351

Background A pilot using point-of-care (POC) technology for men with urethritis symptoms found most patients (80\%) prefer same day results. We designed a new pathway for managing these patients.

Methods Through this pathway, symptomatic men had a nucleic acid amplification test for chlamydia and gonorrhoea (CT/GC NAAT) in clinic, and returned for treatment, usually the same day. Use of Panther (Hologic Inc) at POC provided rapid CT/GC NAAT results within four hours. Microscopy was limited to those testing CT/GC NAAT-negative. GC-culture was sent if GC-positive, when treatment was administered. If CT/GC NAAT-negative and non-gonococcal urethritis (NGU) negative the patient was reassured with information on why they might be experiencing pain (anxiety can increase pelvic floor muscle tone resulting in referred pain and genitourinary symptoms), guided on how to relax their pelvic floor, and advised to re-attend if symptoms persisted.

We compared outcomes (Chi-square) over 6 weeks postimplementation in 2020, to 12 weeks in 2014/15.

Results Of 265 symptomatic men in the new pathway, 33/265 (12.5\%) had CT and 30/265 (11.3\%) GC, similar to rates $(\mathrm{p}>0.5)$ in $2014 / 15$ with $59 / 431(13.7 \%)$ and $45 / 431(10.4 \%)$ respectively. 40/264(15.1\%) GC-culture specimens were sent, compared to $385 / 431(89.3 \%)(\mathrm{p}<0.0001)$ in $2014 / 15$.

With our new pathway, 180/265(67.9\%) proceeded to microscopy, with NGU diagnosed in 61 (23\%) compared to $385 / 431(89.3 \%)(\mathrm{p}<0.0001)$ proceeding to microscopy in 2014/15; with $192 / 431(44.5 \%)(\mathrm{p}<0.0001)$ diagnosed with urethritis; 154 with NGU and 38 with GC. In 2020, 17/265 (6.4\%) were treated for confirmed Mycoplasma genitalium. Total reattendance within 4 weeks of initial presentation was $66 / 265(24.9 \%)$ in 2020 compared to $150 / 431(34.8 \%)$ $(p=0.008)$ in $2014 / 15$.

Conclusions The new rapid service resulted in quicker diagnosis with prompt and specific antimicrobial treatment, reducing the cost and inconvenience of unnecessary microscopy and GC-culture. Patient outcomes and management costs have also improved by reducing reattendance. The new pathway facilitates prompt partner notification, minimising onward STI transmission.

\section{P290 EGOCENTRIC ANALYSIS OF SEXUAL PARTNERSHIPS AND PARTNERSHIP FORMATION IN A MILITARY POPULATION}

${ }^{1,2} \mathrm{~N}$ Rahman*, 1,3R Lugo Robles, 1,2 H Hsieh, 1,2S Waggoner, ${ }^{3} \mathrm{~T}$ Kao, ${ }^{2,3} \mathrm{E}$ Garges. ${ }^{1} \mathrm{Henry}$ M. Jackson Foundation, Bethesda, USA; ${ }^{2}$ Infectious Disease Clinical Research Program, Bethesda, MD; ${ }^{3}$ Department of Preventive Medicine and Biostatistics, Uniformed Services University, Bethesda, USA

\subsection{6/sextrans-2021-sti.352}

Military service provides unique opportunities for sexual partnership development. Changes in social networks, geographic relocation, and other bridging opportunities may contribute to the high burden of sexually transmitted infections (STI) in the military. Here we present a statistical analysis of sexual partnerships in a sample of military beneficiaries at five military treatment facilities.

A sample of 821 military beneficiaries completed a computer-assisted self-interview (CASI) cross-sectional egocentric survey of sexual history and individual STI risk factors and a detailed 90-day sexual partner inventory. Additional demographical and clinical data were captured from the electronic medical record. Weighted logistic regression was used to assess the association between risk factors and laboratory-confirmed STI.

669 of $821(81.49 \%)$ subjects submitted at least one partnership survey, yielding data on 1,416 sexual partnerships. Partnerships per respondent ranged from 0-24. Condom or dental dam usage by partnership type (MSM, MSW, WSM, WSW) ranged from $5-32 \%$ at last sex act, and was associated with partnership status (main/'steady' versus casual/anonymous) 